enzymes may result in neuronal cell damage after febrile seizures. That febrile seizures may be less benign than generally assumed, as suggested by this study, is also apparent from a recent report of MRI abnormalities that followed both simple and complex febrile seizures (Hesdorffer DC et al. Epilepsia 2008;49:765-771), and an increased risk of sudden unexpected death during 2 years after a first febrile seizure (Vestergaard $\mathrm{M}$ et al, Lancet 2008;372:457-463) (Ped Neur Briefs 2008;22:47-48 and 68-69). Recommendations for the management of febrile seizures are outlined in a report of the Italian League Against Epilepsy (Epilepsia Jan 2009;50(Suppl 1):2-6), with reference to the AAP Guidelines 1996, 1999, and to Fukuyama Y et al. Brain Dev 18:479-484. The possible benefits of prolonged antiepileptic therapy to prevent recurrence of febrile seizures must be weighed against the reported adverse cognitive effects. Currently, chronic prophylactic treatment is usually discouraged, except in some patients with recurrent complex febrile seizures. More effective antipyretic treatment, rapid viral testing and antiviral therapy should provide a more specific approach to the prevention of recurrence of febrile seizures (Millichap JJ et al. J Infect Dis 2008;198:1093-4).

\title{
RISK FACTORS FOR NEONATAL SEIZURES
}

The association between maternal characteristics and birth admission seizures in a cohort of 2.3 million California children born at >36 weeks' gestation between 1998 and 2002 was evaluated using database statistics at the University of California San Francisco and Davis, CA. The incidence of birth admission seizures was $0.95 / 1000$ live births. The risk of seizures was higher in male infants. Women age 40 years and older who were nulliparous, had diabetes mellitus, intrapartum fever, or infection (chorioamnionitis) or delivered at $>42$ weeks had an increased risk of delivering an infant with seizures. Maternal/race ethnicity was significantly associated with seizures, with a lower risk in infants of Hispanic and Asian compared with Caucasian and African-American mothers. Identifying antenatal and intrapartum risk factors for neonatal seizures might lower infant neurological morbidity and mortality. Late delivery is one risk factor that might be avoided. (Glass HC, Pham TN, Danielsen B, Towner D, Glidden D, Wu YW. Antenatal and intrapartum risk factors for seizures in term newborns: a population-based study, California 1998-2002. J Pediatr Jan 2009;154:24-28). (Reprints: Hannah C Glass, University of California San Francisco, Department of Neurology Box 0663, 521 Parnassus Ave, C-215, San Francisco, CA 94143. E-mail: Hannah.Glass@ucsf.edu.).

COMMENT. Previous reports found a higher incidence of neonatal seizures, from 1 to 3.5 per 1000 in term infants, but other studies included seizures up to 28 days of life. The increased risk of seizures at birth with maternal intrapartum fever and infection remains elevated even when meningitis or neonatal infection is excluded. The risk is higher in febrile multiparous compared to nulliparous women. A higher risk of seizures in infants born by cesarean section during night hours is unexplained.

Unexplained seizures in an infant. A girl born by cesarean section at 38 weeks after placental detachment developed generalized tonic-clonic seizures and tremors at 2 months. Blood tests, EEG and MRI were normal. The mother was receiving amitriptyline for postnatal depression and tension headache. The infant was treated with phenobarbital but 
seizures recurred, even during hospitalization. Metabolic studies were considered. At one hospital stay, the seizure was complicated by ventricular tachycardia and fibrillation. After transfer to intensive-care for resuscitation, she was comatose and hypotonic, her pupils were dilated but reactive, and she was ventilated mechanically. She rapidly recovered consciousness, breathed independently, and moved all limbs. Mother was unimpressed and denied improvement in her child's condition. Toxicological studies obtained because of mother's incongruous response disclosed serum concentrations of amitryptyline $350 \mathrm{mcg} / \mathrm{L}$. Mother admitted that she had administered amitryptyline drops to the infant for the past month. When removed to live with maternal grandmother, the child remained well and without seizures. The child was a victim of the factitious disorder, Munchausen's syndrome by proxy (Astuto M et al. Lancet Jan 3, 2009;373:94).

\section{MRI FINDINGS AND SEIZURE OUTCOMES COMPARED IN FOCAL CORTICAL DYSPLASIAS TYPES I AND II}

Preoperative clinical, neuropsychological, EEG, and imaging features and surgical outcomes of focal cortical dysplasias (FCD) types I and II were compared in 40 children with refractory epilepsy and histopathologically confirmed FCD subtypes, in a study at Charles University, Prague, Czech Republic and centers in Germany. FCD type I was identified in 24 and FCD type II in 16 patients. Seizure types were secondarily generalized tonic-clonic in $54 \%$ of FCDI and 37\% FCDII; and infantile spasms in $21 \%$ of FCDI and $12 \%$ FCDII patients. Status epilepticus occurred in $4 \%$ of FCDI and $12 \%$ of FCDII patients. Multilobar involvement was found in $92 \%$ patients with FCDI and in $50 \%$ of FCDII patients. FCD type I MRI findings include white matter signal changes on T2 images and/or FLAIR, and reduction of white matter volume. FCD type II MRI showed increased cortical thickness, transmantle sign, gray-white matter junction blurring, fluid-attenuated inversion recovery (FLAIR) and proton density (PD) gray and white matter signal changes. Hippocampal atrophy occurred in only 1 patient of each type. Patients with FCD type I showed EEG continuous slowing more commonly; they had lower IQ levels and more behavioral disorders. Postsurgical seizure freedom occurred in 21\% FCD type I and in 75\% FCD type II patients $(\mathrm{P}<0.001)$. (Krsek $\mathrm{P}$, Pieper $\mathrm{T}$, Karimeier $\mathrm{A}$ et al. Different presurgical characteristics and seizure outcomes in children with focal cortical dysplasia type I or II. Epilepsia Jan 2009;50:125-137). (Respond: Pavel Krsek MD PhD, Department of Pediatric Neurology, Charles University, Second Medical School, Motol University Hospital, V Uvalu 84, CZ 15006 Prague 5, Czech Republic. E-mail:pavel.krsek@post.cz).

COMMENT. FCD is reported in up to $25 \%$ of surgical patients with intractable epilepsy and 5-10\% of epilepsies. FCD Ia and Ib, and FCD IIa and IIb are classified histopathologically. Type IIa is distinguished by dysmorphic, and IIb by balloon cells. FCD type I patients present within the first year of life, and have multilobar involvement, high seizure frequency, more complications, and a worse prognosis than FCD type II patients. FCDI is more frequently associated with mental retardation and behavioral disorders, more difficult to localize by EEG and MRI, and has less favorable surgical outcome. The authors consider surgery except for hemispherectomy is frequently palliative in FCDI cases. FCD type I should be considered a distinct entity. 